\title{
Makine Öğrenmesi Metotları Kullanarak Krom III Kaplama Banyosunun Örtme Gücünün Tahmin Edilmesi
}

\author{
Ramazan Katirci $^{*}$, Hidayet Takçı ${ }^{2}$ \\ ${ }^{1}$ Metalurji ve Malzeme Mühendisliği, Sivas Bilim ve Teknoloji Üniversitesi, Sivas, Turkey \\ ${ }^{2}$ Bilgisayar Mühendisliği, Sivas Cumhuriyet Üniversitesi, Sivas, Turkey \\ ${ }^{* 1}$ ramazankatirci@ sivas.edu.tr, ${ }^{2}$ htakci@ @umhuriyet.edu.tr
}

(Geliş/Received: 10/06/2021;

Kabul/Accepted: 23/08/2021)

\begin{abstract}
Öz: Bu çalışmada krom kaplamanın örtme gücünü tahmin etmek ve örtme gücüne etki eden öznitelikleri belirlemek için makine öğrenmesi algoritmaları kullanılmıştır. Bu amaçla GP (Gaussian Process), KNN (K-Nearest Neighbors), RF (Random Forest), SVR (Support Vector Regressor) ve XGB (eXtreme Gradient Boosting) algoritmaları seçilmiş ve bu algoritmaların hiper parametreleri optimize edilmiştir. En yüksek R2 ve en düşük MSE değerlerini veren şartlar belirlenmiştir. Çapraz doğrulama için LOO (Leave-One-Out) metodu kullanılmıştır. En iyi sonuç, SVR metodu ile elde edilmiştir. R2, MSE ve MAPE değeri sırasıyla 0,80, 0,26 ve 18.29 dur. Kaplamanın örtme gücüne etki eden en önemli iki öznitelik borik asit $\left(\mathrm{H}_{3} \mathrm{BO}_{3}\right)$ ve $\mathrm{A}$ kimyasalıdır. Bu kimyasalların yüksek seviyeleri kaplamanın örtme gücünü artırmıştır. Tüm algoritmaların hiper parametreleri 1zgara tarama yöntemi ile 2 veya daha fazla seviyede optimize edilmiştir. SVR metodunda en etkin iki hiper parametre kernel ve C parametresidir. Kernel ve C hiper parametreleri sırasıyla "rbf" ve 1 olduğu durumda en yüksek R2 değeri elde edilmiştir. $\mathrm{Bu}$ çalışma makine öğrenmesi algoritmalarını elektrokaplama sahasına uygulayan ilk çalışmalardandır. Bu yönüyle öncü olma niteliği taşımaktadır.
\end{abstract}

Anahtar kelimeler: Makine öğrenmesi, Cr(III) Elektrokaplama, Destek Vektör Regresyonu (SVR), Rassal Orman (RF), KEn Yakın Komşu Algoritması (KNN)

\section{Prediction of Covering Power of Chromium III Plating Bath Using Machine Learning Methods}

\begin{abstract}
In this study, machine learning algorithms were used to predict the covering power of chrome plating and to determine the features that affect the covering power. For this purpose, GP (Gaussian Process), KNN (K-Nearest Neighbors), RF (Random Forest), SVR (Support Vector Regressor) and XGB (eXtreme Gradient Boosting) algorithms were selected and their hyper-parameters were optimized. Conditions giving the highest R2 and lowest MSE values were acquired. The LOO (Leave One Out) method was used for cross validation. The best results were obtained using the SVR method. The R2, MSE and MAPE scores are 0.80, 0.26 and 18.29, respectively. The two most important features affecting the the covering power of the coating are boric acid $\left(\mathrm{H}_{3} \mathrm{BO}_{3}\right)$ and chemical A. High levels of these chemicals increased the covering power of the coating. The hyper-parameters of all algorithms were optimized at 2 or more levels by the grid scan method. The two most effective hyper-parameters in the SVR method are the kernel and the $\mathrm{C}$ parameters. The highest R2 value was obtained when the Kernel and C hyper-parameters were "rbf" and 1, respectively. This study is one of the first studies to apply machine learning algorithms to the electroplating field.
\end{abstract}

Key words: Machine learning, Cr(III) Electroplating, Support Vector Regression (SVR), Random Forest (RF), KNearest Neighbor Algorithm (KNN)

\section{Giriş}

Krom kaplama, aşınmaya dayanıklı, korozyon direnci yüksek ve parlak yüzey kaplamalar elde etmek için endüstride yaygın olarak kullanılmaktadır [1]. Krom III ve krom VI (+3 ve +6 değerlikli krom iyonu) tuzlarından elde edilebilmektedir [2]. Krom VI toksik olduğu için hem dünyada hem de ülkemizde yasalarla sınırlandırılmıştır [3]. Krom VI banyosunun bakımı ve işletimi krom III banyosuna göre çok daha kolaydır. Bu yüzden endüstride en fazla krom VI banyosu kullanılmaktadır. Ayrıca krom III banyosunun kararlılığı krom VI ya göre daha düşüktür [4]. Krom III banyosunun sıcaklık, organik katkılar ve metalik kirliliklere karşı duyarlılığı yüksek iken, krom VI banyosunun düşüktür [5-7]. Krom III banyosunun yaygınlaştırılabilmesi için bu dezavantajlarının giderilmesi önemlidir. Krom III banyosunun bakımı ve işletim zorlukları yapay zekâ yöntemleri kullanılarak azaltılabilir. Kaplama banyosunun, kaplama kalitesini tahmin etmek için en sık kullanılan yöntem Hull-hücresinde yapılan kaplamadır [8]. Hull-hücresinde, düşük akım ve yüksek akım yoğunluğu bölgelerinde kaplamanın özellikleri aynı

\footnotetext{
* Sorumlu Yazar: ramazankatirci@ sivas.edu.tr. Yazarların ORCID numarası: : ${ }^{1}$ 0000-0003-2448-011X, ${ }^{2}$ 0000-0002-4448-4284
} 
anda incelenebilmektedir [9]. Bir teknisyen Hull-hücresinde kapladığı plakaya bakarak banyodaki eksiklikleri tahmin edebilmektedir [10]. Aynı tahmini yapay zeka algoritmaları kullanılarak yapmak mümkündür. Lenz ve arkadaşları, 2020 yılında kaplama yapışma gücünü CNN algoritmaları kullanarak tahmin etmişlerdir. Bu test yönteminde kaplama üzerine DIN EN ISO 20502:2016-11 standardına göre farklı yükler uygulanarak çizik atılmış ve oluşan çiziğin derinliğinden ve genişliğinden yapışma gücünü tahmin etmişlerdir [11]. Bu amaçla AlexNet [12], Inception-v3 [13], Resnet-101 [14] ve VGG-19 [15] CNN algoritmaları araştırılmış, VGG-19 modelinin insan tahminine en yakın sonuç verdiği belirtilmiştir. Ayrıca benzer çalışma, Wang ve ekibi tarafından CNN algoritmaları kullanılarak, plazma spray kaplama yönteminde kontrol parametrelerini tahmin etmişlerdir [16]. Katırcı ve ekibi 2021 yılında, çinko-nikel banyosu içeriğinden kaplama kalınlığını ve nikel alaşım oranlarını makine öğrenmesi yöntemleri kullanarak tahmin etmişlerdir. En yüksek R kare $\left(R^{2}\right)$ değerini (kalınlık için $r 2=0,87$, $\% \mathrm{Ni}$ için r2 =0,81) XGBoost makine öğrenmesi metodu kullanarak bulmuşlardır [17].

Bu çalışmada, krom III elektrokaplama banyosu içeriğinden kaplamanın örtme gücü tahmin edilmiştir. Krom kaplama banyosunun örtme gücü, nikel, bakır ve çinko gibi elektrokaplama banyolarına göre çok daha düşüktür ve kontrol edilmesi önemlidir. Elektrokaplama banyosunda örtme gücü, kaplamanın parça üzerinde derin bölgelere girebilme gücünü göstermektedir. Eğer krom elektrokaplama banyosu yeterince örtme gücüne sahip değilse, parçaların iç ve derin bölgeleri kaplama almamakta ve bu bölgeler korozyona maruz kalmaktadır. Ayrıca kaplamanın görselliği bozulmaktadır. Krom kaplama genellikle bakır/nikel üzerine ya da sadece nikel kaplama üzerine uygulanmaktadır. Krom III elektrokaplama banyosunun örtme gücünü tahmin etmek için GP [18], KNN [19], RF [20], SVR [21] ve XGB [22] makine öğrenmesi algoritmaları kullanılmıştır. Her bir makine öğrenmesi algoritmasının hiper parametreleri ızgara tarama yöntemi kullanılarak optimize edilmiştir. Hiper parametreler, modeli optimize etmek için değeri kullanıcı değiştirilebilen model parametreleridir. Algoritmaların doğruluğu LOO [23] yöntemi kullanılarak test edilmiştir. Ayrıca özniteliklerin (girdilerin) ağırlıklarını tespit etmek için XGB ve RF makine öğrenmesi algoritmaları kullanılmış ve bulunan sonuçlar kıyaslanmıştır.

\section{Deneysel Yöntem}

Krom kaplama, Thurlby 30V1A-model DC model doğru akım kaynağı kullanılarak yapılmıştır. Deney deseni 1/16 kesirli deneysel tasarım metodu [24] kullanılarak hazırlanmıştır. Deney deseninde Tablo 1'deki değerler kullanılmıştır. Toplam 16 farklı elektrokaplama banyosu hazırlanmış ve her bir banyoda ardı ardına 3 plaka kaplanmıştır. Toplamda 48 kaplanmış plaka elde edilmiştir. Kaplamanın örtme gücü Şekil 1'deki "1” mesafesi ölçülerek elde edilmiştir. "1" mesafesi değeri ne kadar düşükse kaplamanın örtme gücü o kadar iyi anlamına gelmektedir. $\mathrm{H}_{3} \mathrm{BO}_{3}, \mathrm{pH}$ '1 dengede tutmak için sodyum sülfat $\left(\mathrm{Na}_{2} \mathrm{SO}_{4}\right)$ ise çözeltinin elektriksel iletkenliğini arttırmak için kullanılmıştır. Diğer organik ve inorganik katkı maddeleri ticari sebeplerden dolayı A, B, C, D, E olarak kodlanmıştır. Tüm kaplamalar Hull hücresinde gerçekleştirilmiştir. Krom kaplama için Şekil 2'deki Hull hücresi kullanılmıştır.

Tablo 1. Deneysel tasarım metodunda kullanılan kimyasallar ve miktarları.

\begin{tabular}{lll}
\hline Kimyasallar & Seviye 1 & Seviye 2 \\
\hline A & $100 \mathrm{ml} / \mathrm{L}$ & $200 \mathrm{ml} / \mathrm{L}$ \\
B & $20 \mathrm{ml} / \mathrm{L}$ & $40 \mathrm{ml} / \mathrm{L}$ \\
C & 0 & $3 \mathrm{~g} / \mathrm{L}$ \\
D & 0 & $1 \mathrm{~g} / \mathrm{L}$ \\
E & 0 & $12,5 \mathrm{ml} / \mathrm{L}$ \\
pH & 3 & 3,5 \\
Sodyum sülfat $\left(\mathrm{Na}_{2} \mathrm{SO}_{4}\right)$ & $100 \mathrm{~g} / \mathrm{L}$ & $150 \mathrm{~g} / \mathrm{L}$ \\
Borik asit $\left(\mathrm{H}_{3} \mathrm{BO}_{3}\right)$ & $40 \mathrm{~g} / \mathrm{L}$ & $60 \mathrm{~g} / \mathrm{L}$ \\
\hline
\end{tabular}




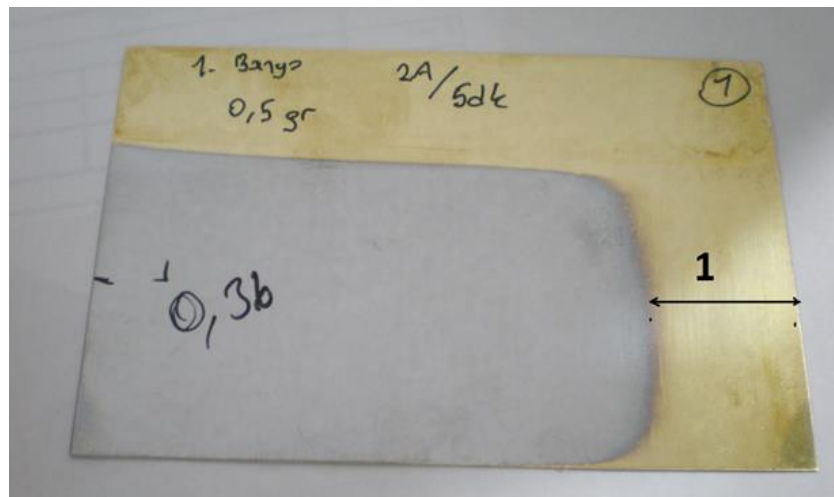

Şekil 1. Cr kaplı pirinç plaka: 1 mesafesi kaplamanın örtme gücünü gösteren bölgedir.

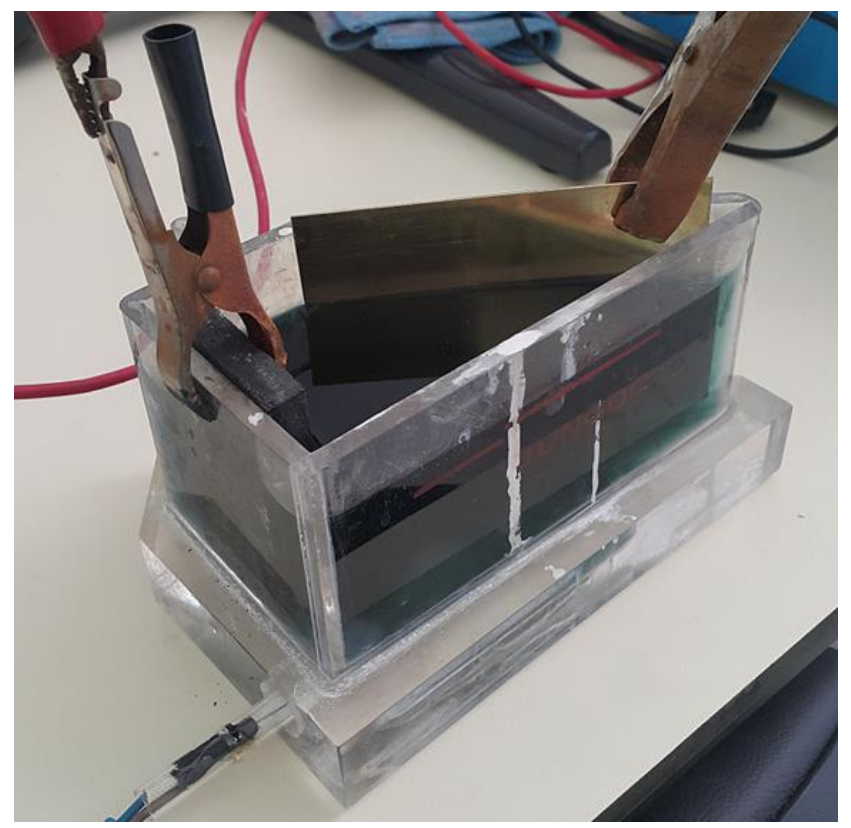

Şekil 2. Cr (III) elektrokaplama düzeneği (Hull-hücresi).

\section{3. Öğrenme Algoritmaları}

Makine öğrenmesi metotları son yıllarda mühendislik alanlarında yaygın olarak kullanılmaktadır [25-27]. Hem regresyon hem de sınıflandırma verilerinde tercih edilmektedir. Çalışmamızda, veriler sürekli yapıda olduğundan regresyon algoritmaları kullanılmıştır. Verisetindeki örnek sayısı az olduğundan çapraz doğrulama olarak LOO yöntemi tercih edilmiştir [28]. Bu yöntemin en önemli avantajlarından biri test hata değerinin her zaman her aynı değeri vermesidir. Diğer yöntemlerde "eğitim" ve "test" verisetlerindeki örnekler rastgele seçildiğinden test hata değerleri değişmektedir. LOO yönteminde her bir veri hem test hem de eğitim verisi olarak kullanılmaktadır. Birinci model eğitimi için toplam 48 veriden birincisi test verisi olarak ayrılır, diğer geri kalan 47 veri eğitime tabi tutulur. Eğitim bittikten sonra test olarak ayrılan veri tahmin edilir. İkinci model eğitiminde ise ikinci veri, test verisi olarak ayrılır diğer geri kalan veri eğitime tabi tutulur. Eğitim sonrası test verisi tahmin edilir. Toplam 48 veri için 48 model eğitilir ve 48 veri tahmin edilir. Dolayısı veri sırasının rastgele seçilmesi test hata değerini etkilemez. Eğitim ve tahmin için GP, KNN, RF, SVR ve XGB makine öğrenmesi algoritmaları kullanılmış, her bir algoritmanın hiper parametreleri optimize edilmiştir. Elde edilen sonuçlar en küçük kareler $\left(\mathrm{R}^{2}\right)$ ve ortalama kare hata (MSE) yöntemleri kullanılarak değerlendirilmiştir [29]. $\mathrm{R}^{2}$ ve MSE değerleri Eşitlik 1 ve 2 ye göre hesaplanmıştır. 
$R^{2}=\frac{\sum_{i=1}^{n}\left(Y_{i}-\bar{Y}\right)^{2}-\sum_{i=1}^{n} e_{i}^{2}}{\sum_{i=1}^{n}\left(Y_{i}-\bar{Y}\right)^{2}}$

$Y i$ : Y gerçek değerlerini göstermektedir.

$\bar{Y}:$ Yi değerlerinin ortalama değerlerini göstermektedir.

$e_{i}:$ Y gerçek değeleri ile tahmin değerleri arasındaki farkı göstermektedir.

$$
M S E=\frac{1}{n} \sum_{j=1}^{n} e_{j}^{2}
$$

MSE (Mean Square Error): Ortalama kare hata

$e_{j}$ : Gerçek değerle tahmin değeri arasındaki farkı göstermektedir.

$n$ : Örnek sayısına karşı1ık gelmektedir.

$G P$

GP makine öğrenimi alanında, parametrik olmayan, Bayesian yaklaşımını kullanan bir metottur. GP küçük veri kümeleri üzerinde iyi çalışması ve tahminler üzerinde belirsizlik ölçümleri sağlama becerisine sahip olması önemli avantajlarındandır [18].

\section{$K N N$}

KNN algoritması, gözetimli öğrenme algoritmalarındandır. Hem sınıflandırma hem de regresyon problemlerinin çözümünde kullanılmaktadır. Bu yöntem 1967 yılında T.M. Cover ve P.E. Hart tarafindan önerilmiştir. Algoritma, herhangi bir verinin hangi sınıfa dahil edileceğini k sayıda yakın komşunun mesafesine bakarak bulur [19].

$R F$

2001 yılında Leo Breiman tarafından önerilmiştir. Hızlı çalışır. Hem sınıflandırma hem de regresyon problemlerinde kullanılabilir. Birden fazla karar ağacı üreterek doğruluk değerini yükseltmeyi amaçlayan algoritmadır. Bu modelin diğer özelliklerinden birisi de özniteliklerin sonuca ne kadar katkı sağladığını belirtmesidir [20].

SVR

Destek Vektör Regresyonu (SVR), Vapnik tarafından 1995 yılında önerilen bir algoritmadır. Sürekli yapıya sahip veriler için uygun bir modeldir. Belirli parametreler dikkate alınarak regresyon eğrisi oluşturulmaktadır. Örnek olarak regresyona dahil edilecek verilerin belirli epsilon uzaklıkta olması gerekmektedir [21].

\section{$X G B$}

Tianqi Chen ve Carlos Guestrin, 2016 yılında "A Scalable Tree Boosting System" başlıklı makale ile bu yöntemi duyurmuşlardır. Bu yöntemin en önemli avantajları yüksek tahmin gücüne sahip olması, aşırı öğrenmeyi engellemesi ve boş verilerle çalışabilmesidir. Yazılım ve donanım optimizasyon teknikleri uygulandığından daha hızlı çalışabilmektedir. Hem sınıflandırma hem de regresyon problemlerinde tercih edilmektedir. Ayrıca özniteliklerin ağırlığını tespit etmek için kullanılabilmektedir [22].

\section{Sonuçlar ve tartışma}

Eğitim için kullanılan makine öğrenmesi algoritmalarının hiper parametreleri optimize edildikten sonra en yüksek R2 ve en düşük MSE değerleri tespit edilmiştir. Sonuçlar Tablo 2 de sunulmuştur. Sonuçların güvenirliliğini ve doğruluğunu belirlemek için iki metot kullanılmıştır. Birincisinde verilerin tamamı eğitime dahil edilmiştir. İkincisinde ise LOO çapraz doğrulama yöntemi kullanılmıştır. Hesaplanan R2 ve MSE değerleri 
yorumlanmıştır. Tablo 2'ye göre en iyi sonuç veren makine öğrenmesi algoritması SVR dir. Ancak diğer algoritmalar da (RF, KNN) yakın değerler vermiştir. R2-FULL değeri ile R2-LOO değeri arasındaki fark bize modelin veriyi ne kadar iyi temsil ettiği hakkında bilgi vermektedir. R2-FULL'de tüm veriler eğitime dahil edilmektedir. Eğitim için kullanılan veriler algoritma tarafından tahmin edilmektedir. Dolayısıyla tüm veriler eğitime dahil edildiği için R2 değerleri yüksek çıkmaktadır. R2-LOO'da ise toplam veriden, biri çıkartılmaktadır ve çıkartılan veri algoritma tarafından tahmin edilmektedir. Tüm veriler için aynı durum tekrarlanmaktadır. Buradaki R2 değeri ise her zaman R2-FULL den düşüktür. R2-LOO'da tahmin edilen değer gerçek değere ne kadar yakınsa, eğitilen model, sistemi o kadar iyi temsil ediyor demektir [29]. Yani sistem öğrenmiş, ezber yapmamış demektir. Bu yöntemde tahmin edilen verilerin gerçek değerlerinin bilinmesi gerekmektedir.

Eğitilen modelin tahmin etme gücünü anlamanın diğer bir yolu da R2-FULL ve R2-LOO değerlerinin karşılaştırılmasıdır. R2-FULL de eğitime dahil edilen veriler tahmin edildiği için, eğitime dahil edilmeyen verileri tahmin etme gücü bilinmemektedir. Bu yüzden R2-LOO değerleri hesaplanarak eğitime dahil edilmeyen veriler tahmin edilmektedir. R2-LOO dan elde edilen R2 değeri ve R2-FULL den elde edilen R2 değerine ne kadar yakınsa, eğitilen modelin bilinmeyen verileri tahmin etme gücü o kadar yüksektir denir. Bu açıdan Tablo 2 yeniden incelendiğinde SVR algoritmasının en iyi öğrenmeyi gerçekleştiren model olduğu görülmektedir.

MAPE değerleri Eşitlik 3' göre hesaplanmıştır. Ortalama mutlak yüzde hata (MAPE), istatistikte bir tahmin yönteminin doğruluğunun bir ölçüsüdür. \%10'a kadar olan değerleri çok iyi, \%10-20 arasındaki MAPE değerleri iyi, \%20-50 arasındaki değerler kabul edilebilir, \%50'nin üzerindeki ortalama mutlak yüzde hata değerleri ise yanlıș veya hatalı olarak kabul edilmektedir [30]. Bu kapsamda Tablo 2 incelendiğinde GP modeli hariç diğer modellerin MAPE değerleri \%10-20 arasında çıkmıştır. Dolayısıyla elde edilen sonuçların doğruluk değeri iyi olarak sınıflandırılabilir.

$$
M A P E=\frac{100}{n} \sum_{j}^{n} \frac{\left|e_{j}\right|}{\left|A_{j}\right|}
$$

$\mathrm{e}_{\mathrm{j}}$ : Gerçek değerle tahmin değeri arasındaki farkı göstermektedir.

$\mathrm{A}_{\mathrm{j}}$ : Gerçek değerleri göstermektedir.

$\mathrm{n}$ : Örnek sayısına karşılık gelmektedir.

Tablo 2. Makine öğrenmesi algoritmalarının R2 ve MSE değerleri.

\begin{tabular}{clcccc}
\hline $\begin{array}{c}\text { Öğgrenme } \\
\text { Algoritmaları }\end{array}$ & R2-FULL & MSE-FULL & R2-LOO & MSE-LOO & MAPE-LOO \\
\hline GP & 0.89 & 0.19 & 0.75 & 0.35 & 23.17 \\
KNN & 1 & 0 & 0.78 & 0.25 & 17.41 \\
RF & 0.92 & 0.10 & 0.79 & 0.24 & 17.98 \\
SVR & 0.92 & 0.12 & 0.80 & 0.26 & 18.29 \\
XGB & 0.95 & 0.06 & 0.76 & 0.28 & 19.06 \\
\hline
\end{tabular}

R2-FULL ve MSE-FULL: R2 ve MSE değerleri hesaplanırken, tahmin edilen veri, ĕgitime dahil edilmiştir. R2-LOO ve MSE-LOO: R2 ve MSE değerleri hesaplanırken, tahmin edilen veri, ĕgitime dahil edilmemiştir.

Şekil 3 deneysel sonuçlarla tahmin sonuçlarının karşılaştırmasını göstermektedir. Deneysel verilerdeki ani değişimleri hiçbir model doğru tahmin edememiştir. Özellikle 5. ve 24. verilerde bu net şekilde görülmektedir. Ancak bu veriler dişındaki diğer verilerde yüksek uyum gözlenmektedir. 


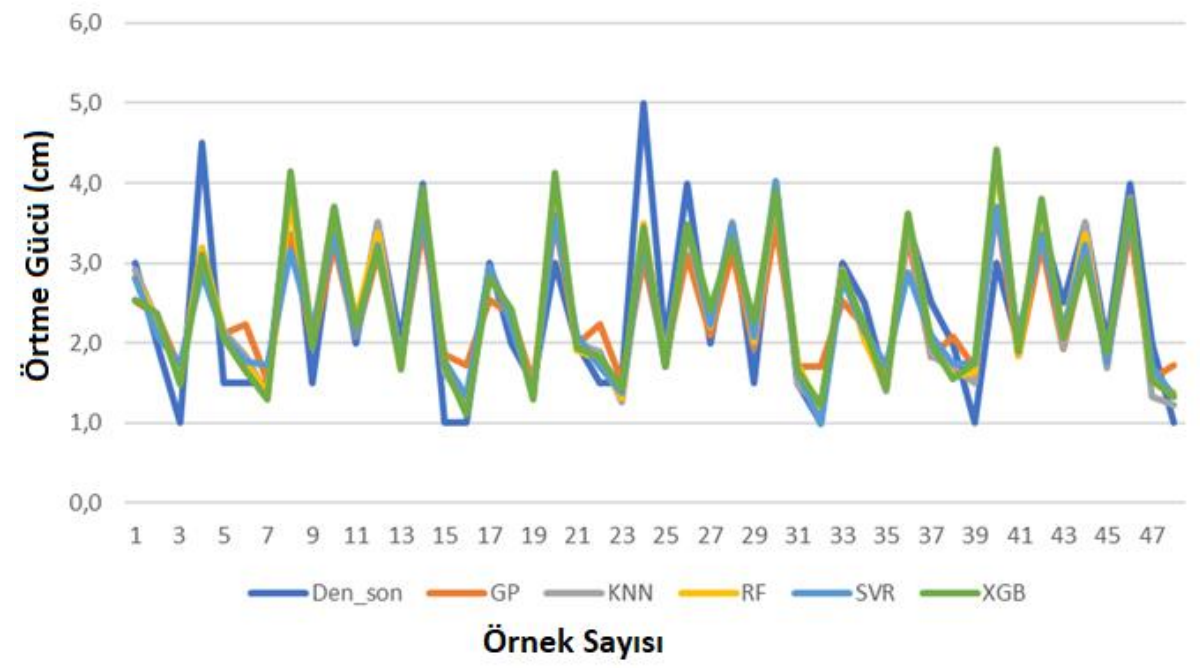

Şekil 3. Deneysel sonuçlarla makine öğrenmesi algoritmalarından elde edilen tahmin sonuçları (LOO çapraz doğrulama metodu gösterilmektedir).

Krom kaplamanın örtme gücünü belirlemede kullanılan faktörlerin (özniteliklerin) ağırlıklarını belirlemek için XGBoost ve RF algoritmaları kullanılmıştır. Bu sayede girdilerden hangilerinin sonuca daha fazla katkı sağladığı ve ne yönde katkı sağladığı belirlenmiştir. Sonuçlar Tablo 3 de gösterilmiştir. Özniteliklerin ağırlıkları ve sırası her iki yöntemde de birbirine yakın çıkmıștır. PNO, aynı banyoda ardı ardına çekilen 3 plakayı göstermektedir. Elektrokaplama banyosunun kararlılı̆̆ hakkında bilgi verir. En düşük ağırlığa sahiptir. Bu bize banyo kararlılı̆̆ının yüksek olduğunu göstermektedir. Örtme gücünü en çok etkileyen özniteliklerin $\mathrm{H}_{3} \mathrm{BO}_{3}$ ve $\mathrm{A}$ malzemesi olduğu görülmektedir. A malzemesi Cr (III) iyonları içermektedir. Banyo içindeki Cr (III) iyonları miktarı arttıkça kaplamanın örtme gücüde ilerlemektedir. Aynı durum $\mathrm{H}_{3} \mathrm{BO}_{3}$ kimyasalı içinde gözlenmiştir. C, D ve E kimyasalları krom +3 iyonlarının krom metaline daha kolay indirgenebilmesi için kullanılan kompleksleyici ajanlardır. Bunların miktarının da krom kaplamanın örtme gücünü önemli ölçüde etkilediği gözlenmiştir. C ve $\mathrm{E}$ kimyasalları D kimyasalına göre daha etkindir.

Tablo 3. Özniteliklerin ağırlıkları

\begin{tabular}{lcc}
\hline Öznitelikler & \multicolumn{1}{c}{ XGB } & RF \\
\hline PNO & 0,03 & 0,01 \\
A & 0,23 & 0,26 \\
B & 0,05 & 0,06 \\
C & 0,11 & 0,11 \\
D & 0,05 & 0,05 \\
E & 0,10 & 0,11 \\
pH & 0,08 & 0,07 \\
$\mathrm{Na}_{2} \mathrm{SO}_{4}$ & 0,05 & 0,04 \\
$\mathrm{H}_{3} \mathrm{BO}_{3}$ & 0,29 & 0,29 \\
\hline
\end{tabular}

Bu çalışmada tüm algoritmaların hiper parametreleri en yüksek R2 ve en düşük MSE değerini verecek şekilde optimize edilmiştir. SVR metodu en yüksek R2 değerini verdiği için bu bölümde SVR algoritmasının hiper parametreleri tartışılmıştır. Optimize edilmiş hiper parametreler ve değerleri Tablo 4'de verilmiştir. Hiper parametrelerin etkinliği ve birbiriyle etkileşimi Şekil 4-7'de gösterilmiştir. Şekil 4 incelendiğinde R2'yi etkileyen en etkin hiper parametrelerin kernel ve $\mathrm{C}$ olduğu görülmektedir. C bir düzeltme parametresidir. Kernel ise uygulanacak optimizasyon algoritması tipini göstermektedir. R2 değerinin en yüksek olduğu kernel yapısı linear 
(lineer) olarak görülmektedir. Ancak Tablo 4 incelendiğinde en yüksek R2 değeri kernelin rbf (radial basis function)[30] olduğu durumda gözlenmiştir. Bu çelişki bize kernel ile diğer bir hiper parametre arasında etkileşim olduğunu göstermektedir. Bunun için Şekil 5'in incelenmesi önemlidir. Şekil 5 de kernelin C parametresi ile etkileşim halinde olduğu görülmektedir. C parametresi 0 ve 5 olduğunda rbf kerneli en düşük R2 değerini, 1 olduğunda ise "rbf" parametresi en yüksek R2 değerini vermektedir. Degree parametre değeri yükseldikçe R2 değeri düşmektedir. Degree parametresi sadece kernel in poly (polynomial) seçildiği durumda geçerlidir. Diğer durumlarda degree parametresi ihmal edilmektedir. Bu yüzden rbf kernel inde degree parametresinin bir anlamı yoktur. Cach_size hiper parametresinin optimizasyon aşamasında hiçbir etkinliği görülmemiştir. Gamma parametresi düşük değerlerde daha yüksek R2 değeri vermiştir. Bu parametre kernel katsayısını ifade etmektedir.

Tablo 4. SVR metodunda optimize edilen hiper parametreler.

\begin{tabular}{ll}
\hline Hiper parametreler & Değerler \\
\hline $\mathrm{C}$ & 1 \\
cache_size & 10 \\
degree & 1 \\
epsilon & 0,1 \\
gamma & 0,1 \\
kernel & 'rbf' \\
\hline
\end{tabular}

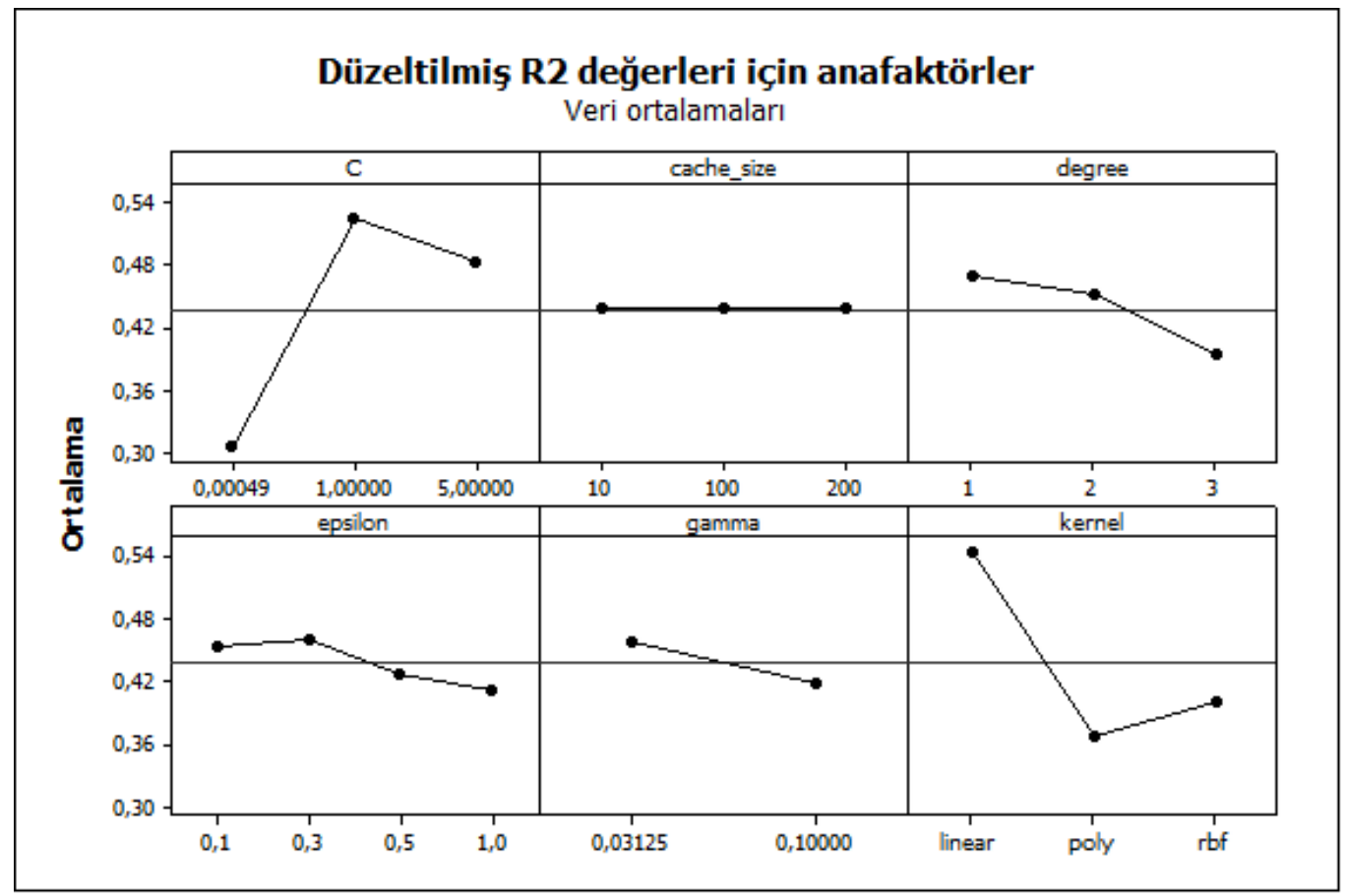

Şekil 4. Hiper parametrelerin R2 değerine etkisi (Dikey eksen ortalama R2 değerlerini, yatay eksen ise hiper parametrelerin aldığ 1 değerleri göstermektedir). 


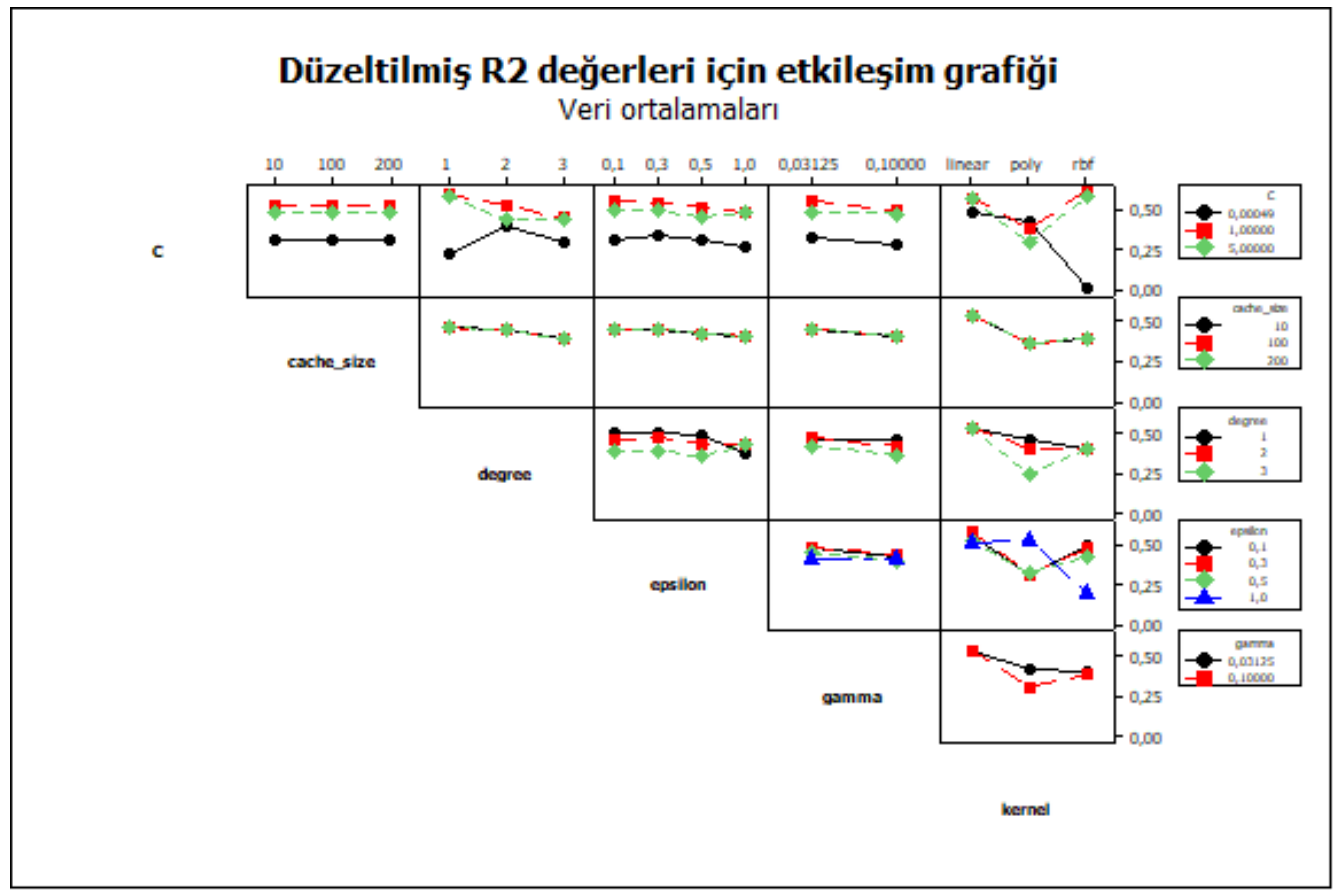

Şekil 5. Hiper parametrelerin R2 değerine etkisini gösteren ikili etkileşim grafiği (Dikey eksen ortalama R2 değerlerini, yatay eksen ise hiper parametrelerin aldığı değerleri göstermektedir).

MSE değerini düşüren en etkin hiper parametreler ise şekil 6'da gösterilmiştir. Epsilon, kernel ve degree hiper parametreleri en etkin hiper parametreler olarak görülmektedir. Şekil 6'ya göre epsilon değeri 1 olduğu durumda en düşük MSE değeri elde edilmiștir. Ancak Tablo 4'e göre en optimum değer 0.1 olduğu durumda elde edilmiștir. $\mathrm{Bu}$ sonuç bize epsilon değeri ile başka bir hiper parametrenin etkileşim yaptığını göstermektedir. Şekil 7 bize rbf kernel inde ve epsilon 1 değerinde MSE değerinin en yüksek değere sahip olduğunu göstermektedir. Epsilon 0,1 değerinde ise en düşük MSE değeri görülmektedir. Gamma hiper parametresi düşük değerde daha düşük MSE ve daha yüksek R2 değeri vermesine rağmen Tablo 4'te yüksek değer tercih edilmiştir. Bunun sebebini anlamak için Şekil 7'deki etkileşim grafiğine bakmak gerekir. Şekil incelendiğinde gamma hiper parametresinin rbf kernel inde en düşük MSE değeri verdiği görülmektedir. 


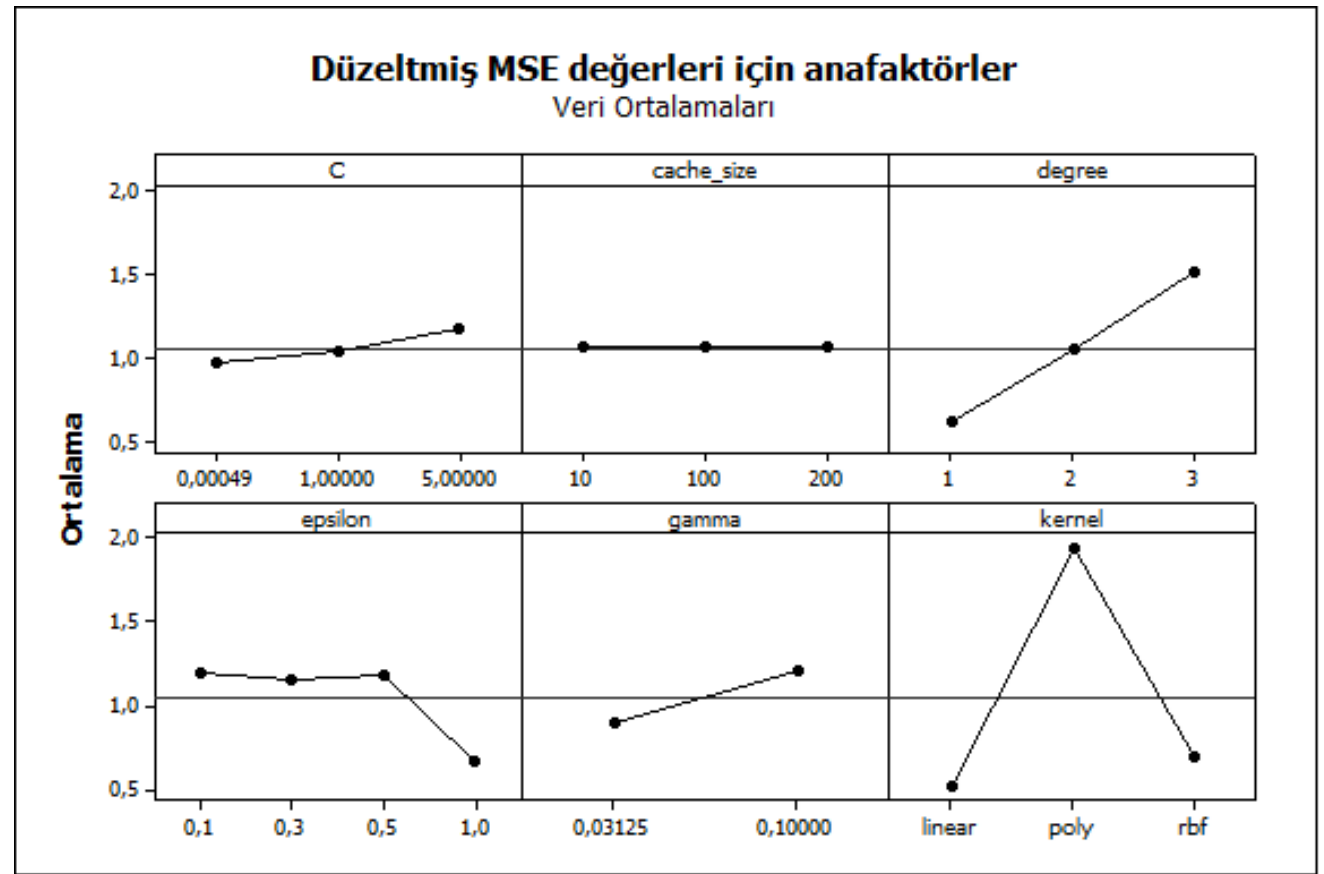

Şekil 6. Hiper parametrelerin MSE değerine etkisi (Dikey eksen ortalama MSE değerlerini, yatay eksen ise hiper parametrelerin aldığı değerleri göstermektedir).

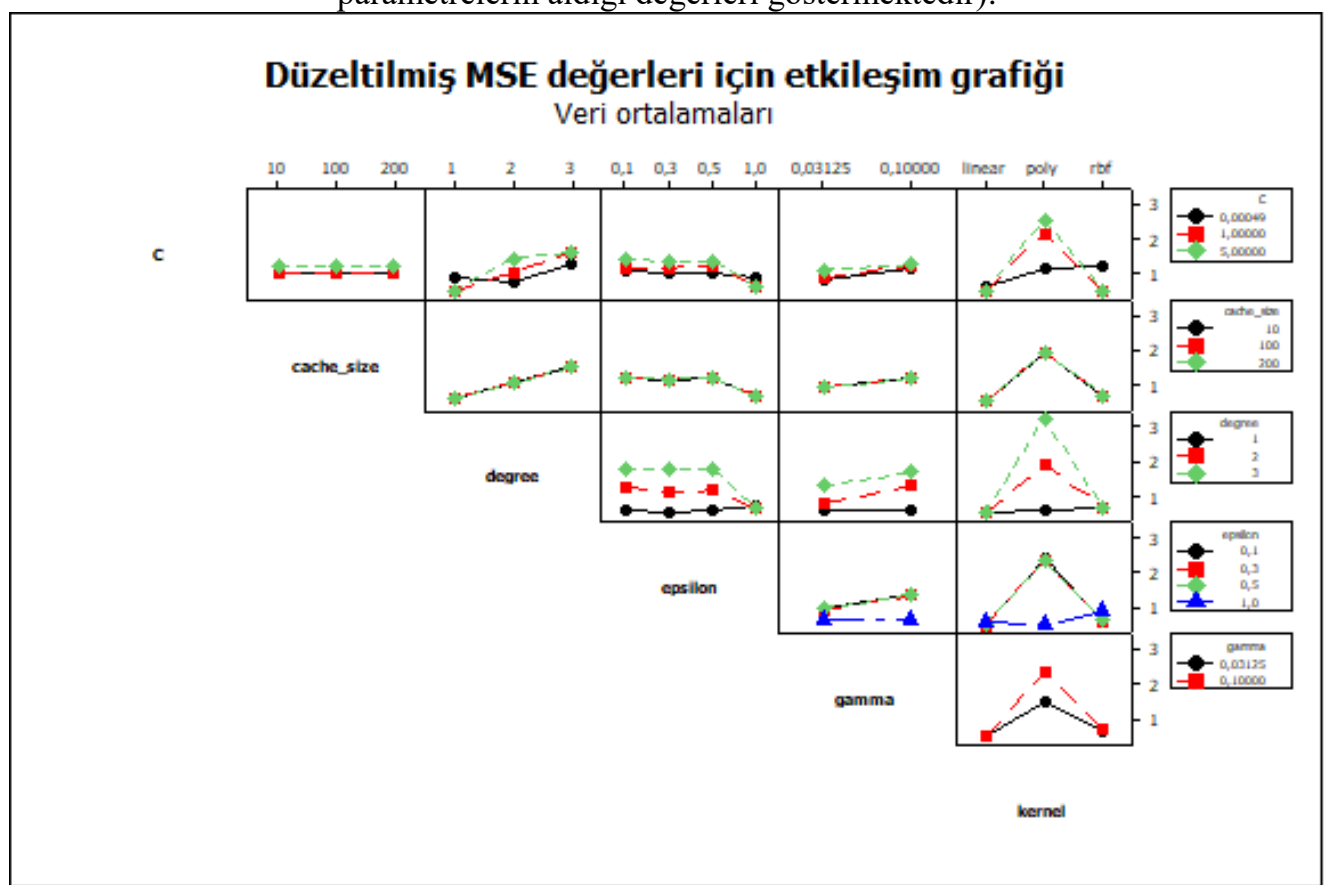

Şekil 7. Hiper parametrelerin MSE değerine etkisini gösteren ikili etkileşim grafiği (Dikey eksen ortalama MSE değerlerini, yatay eksen ise hiper parametrelerin aldığı değerleri göstermektedir).

\section{Değerlendirme}

Sonuç olarak, bu çalışmada makine öğrenmesi algoritmaları kullanılarak Cr (III) kaplama banyosunun örtme gücü araştırılmıştır. Farklı kompozisyonlarda Cr (III) kaplama banyosu hazırlanarak pirinç üzerine krom kaplamalar yapılmıştır. Elde edilen kaplamalardan, kaplama banyolarının örtme gücü tahminlenmeye çalışılmış̧ır. $\mathrm{Bu}$ amaçla 5 makine öğrenmesi algoritması kullanılmıştır. Bunlar GP, KNN, RF, SVR ve XGB dir. En iyi sonuç veren algoritma SVR'dir. R2 ve MSE değerleri sırasıyla 0,80 ve 0,26 'dır. En yakın sonuç veren diğer makine 
öğrenmesi algoritması ise RF'dir. MAPE değerleri \%20'nin altında olduğu için elde edilen sonuçlar güvenilir olarak değerlendirilmiştir.

XGB ve RF algoritmaları kullanılarak öznitelik ağırlıkları hesaplanmıştır. Her iki yöntemle de A kimyasalı ve $\mathrm{H}_{3} \mathrm{BO}_{3}$ en önemli iki öznitelik olarak bulunmuştur. $\mathrm{H}_{3} \mathrm{BO}_{3} 40$ ve $60 \mathrm{gr} / \mathrm{L}$ olarak iki farklı seviyede kullanılmıştır. Yüksek miktarda kullanıldığında kaplama banyosunun örtme gücünün daha iyi olduğu görülmüştür. A kimyasalı $\mathrm{Cr}$ (III) iyonları içermektedir. Cr (III) iyonu miktarı arttıkça örtme gücünün arttığı gözlenmiştir. Bu beklenen bir durumdur. En etkin kompleksan ajanları ise $\mathrm{C}$ ve $\mathrm{E}$ kimyasallarıdır.

En yüksek R2 ve en düşük MSE değerlerini bulabilmek için hiper parametrelerin optimizasyonu önemlidir. Bunun için her bir parametre 2 veya daha fazla seviyede denenmiş ve en optimum değeri veren hiper parametreler belirlenmiştir. Tüm algoritmaların optimum değerleri burada verilmemiş, sadece en yüksek R2 değerini veren SVR metodunun optimum değerleri sunulmuştur. Kernel ve C parametresi en önemli iki hiper parametre olarak tespit edilmiştir. Ayrıca bu iki hiper parametre birbiriyle etkileşim yapmaktadır. Bu yüzden anafaktör ve etkileşim grafiği birlikte incelenmelidir.

$\mathrm{Bu}$ çalışmanın temel amacı, makine öğrenmesi algoritmalarını elektrokaplama proseslerine uygulamak ve daha kararlı, sürdürülebilir kaliteli kaplamalar elde etmektir. Elektrokaplama banyolarının kontrolü ve takibi oldukça zordur. Çok fazla kontrol edilmesi gereken parametre mevcuttur. Bunlardan bazıları ölçülebilir (sıcaklık, akım vs.) bazıları da ölçülemezdir. Özellikle çalışan banyolarda şartlar çok dinamik olduğu için banyo içinde kompozisyonlar sürekli değişmektedir. Yeni tür organik ve inorganik safsızlıklar oluşmaktadır. Bunlar kaplamanın hem örtme gücünü hem de kalitesini düşürmektedir. Yapay zekâ yöntemleri bu parametreleri kontrol etmek için önemli bir araç olarak görülmektedir. Bu çalışma ileriki çalışmalar için yol gösterici olacaktır. Mevcut durumda teknisyenler tarafından, kaplanmış hull plakalarına bakılarak banyo içindeki eksiklikler tahmin edilebilmektedir. Aynı tahminleme işlemi $\mathrm{CNN}$ ve RCNN algoritmaları gibi görüntü işleyen algoritmalar tarafından da yapılabilmesi mümkündür.

\section{Teşekkür}

Bu araştırmada yer alan kısmi nümerik hesaplamalar TÜBİTAK ULAKBİM, Yüksek Başarım ve Grid Hesaplama Merkezi'nde (TRUBA kaynaklarında) gerçekleştirilmiştir.

\section{Kaynaklar}

[1] Z. Zeng, Y. Sun, J. Zhang, The electrochemical reduction mechanism of trivalent chromium in the presence of formic acid, Electrochem. Commun. 11 (2009) 331-334. https://doi.org/10.1016/J.ELECOM.2008.11.055.

[2] S. Surviliene, O. Nivinskiene, A. Češuniene, A. Selskis, Effect of $\mathrm{Cr}(\mathrm{III})$ solution chemistry on electrodeposition of chromium, J. Appl. Electrochem. 36 (2006) 649-654. https://doi.org/10.1007/s10800-005-9105-8.

[3] J.-Y. Lee, M. Kim, S.-C. Kwon, Effect of polyethylene glycol on electrochemically deposited trivalent chromium layers, Trans. Nonferrous Met. Soc. China. 19 (2009) 819-823. https://doi.org/10.1016/S1003-6326(08)60357-X.

[4] S.L. Handy, C.F. Oduoza, T. Pearson, Theoretical aspects of electrodeposition of decorative chromium from trivalent electrolytes and corrosion rate study of different nickel/chromium coatings, Trans. Inst. Met. Finish. 84 (2006) 300308. https://doi.org/10.1179/174591906X162946.

[5] X. Ren, Y. Song, A. Liu, J. Zhang, G. Yuan, P. Yang, J. Zhang, M. An, D. Matera, G. Wu, Computational Chemistry and Electrochemical Studies of Adsorption Behavior of Organic Additives during Gold Deposition in Cyanide-free Electrolytes, Electrochim. Acta. 176 (2015) 10-17. https://doi.org/10.1016/j.electacta.2015.06.147.

[6] Z. Feng, A. Liu, L. Ren, J. Zhang, P. Yang, M. An, Computational Chemistry and Electrochemical Mechanism Studies of Auxiliary Complexing Agents Used for Zn-Ni Electroplating in the 5-5'-Diethylhydantoin Electrolyte, J. Electrochem. Soc. 163 (2016) D764-D773. https://doi.org/10.1149/2.0591614jes.

[7] R. Katırc1, E. Sezer, B. Ustamehmetoğlu, Statistical optimisation of organic additives for maximum brightness and brightener analysis in a nickel electroplating bath, Trans. IMF. 93 (2015) 89-96. https://doi.org/10.1179/0020296714Z.000000000219.

[8] K. Sasaki, G.C. Kabushiki, C. Abstracts, P.E.E. Waddell, United States Patent [ 191, (1976) 575-585.

[9] R. Katırc1, A chrome coating from a trivalent chromium bath containing extremely low concentration of $\mathrm{Cr}<\mathrm{SUP}$ align="right">3+</SUP> ions, Int. J. Surf. Sci. Eng. 10 (2016) 73. https://doi.org/10.1504/IJSURFSE.2016.075318.

[10] H.B. Muralidhara, Y. Arthoba Naik, Electrochemical deposition of nanocrystalline zinc on steel substrate from acid zincate bath, Surf. Coatings Technol. 202 (2008) 3403-3412. https://doi.org/10.1016/j.surfcoat.2007.12.012.

[11] B. Lenz, H. Hasselbruch, H. Großmann, A. Mehner, Application of CNN networks for an automatic determination of critical loads in scratch tests on a-C:H:W coatings, Surf. Coatings Technol. 393 (2020) 125764. https://doi.org/10.1016/j.surfcoat.2020.125764.

[12] A. Krizhevsky, I. Sutskever, G.E. Hinton, ImageNet classification with deep convolutional neural networks, Commun. ACM. 60 (2017) 84-90. https://doi.org/10.1145/3065386. 
[13] C. Szegedy, V. Vanhoucke, S. Ioffe, J. Shlens, Z. Wojna, Rethinking the Inception Architecture for Computer Vision, Proc. IEEE Comput. Soc. Conf. Comput. Vis. Pattern Recognit. 2016-Decem (2016) 2818-2826. https://doi.org/10.1109/CVPR.2016.308.

[14] K. He, X. Zhang, S. Ren, J. Sun, Deep residual learning for image recognition, Proc. IEEE Comput. Soc. Conf. Comput. Vis. Pattern Recognit. 2016-Decem (2016) 770-778. https://doi.org/10.1109/CVPR.2016.90.

[15] K. Simonyan, A. Zisserman, Very deep convolutional networks for large-scale image recognition, 3rd Int. Conf. Learn. Represent. ICLR 2015 - Conf. Track Proc. (2015) 1-14.

[16] J. Zhu, X. Wang, L. Kou, L. Zheng, H. Zhang, Prediction of control parameters corresponding to in-flight particles in atmospheric plasma spray employing convolutional neural networks, Surf. Coatings Technol. 394 (2020) 125862. https://doi.org/10.1016/j.surfcoat.2020.125862.

[17] R. Katirci, H. Aktas, M. Zontul, The prediction of the $\mathrm{ZnNi}$ thickness and $\mathrm{Ni} \%$ of $\mathrm{ZnNi}$ alloy electroplating using a machine learning method, Trans. Inst. Met. Finish. 99 (2021) 162-168. https://doi.org/10.1080/00202967.2021.1898183.

[18] C.E. Rasmussen, C.K.I. Williams, Gaussian Processes for Machine Learning (Adaptive Computation and Machine Learning), The MIT Press, 2005.

[19] L. Peterson, K-nearest neighbor, Scholarpedia. 4 (2009) 1883. https://doi.org/10.4249/scholarpedia.1883.

[20] Y.L. Pavlov, Random forests, Random For. (2019) 1-122. https://doi.org/10.1201/9780429469275-8.

[21] C. Cortes, V. Vapnik, Support-vector networks, Mach. Learn. 20 (1995) 273-297. https://doi.org/10.1007/BF00994018.

[22] T. Chen, C. Guestrin, XGBoost, in: Proc. 22nd ACM SIGKDD Int. Conf. Knowl. Discov. Data Min., ACM, New York, NY, USA, 2016: pp. 785-794. https://doi.org/10.1145/2939672.2939785.

[23] G.I. Webb, C. Sammut, C. Perlich, T. Horváth, S. Wrobel, K.B. Korb, W.S. Noble, C. Leslie, M.G. Lagoudakis, N. Quadrianto, W.L. Buntine, N. Quadrianto, W.L. Buntine, L. Getoor, G. Namata, L. Getoor, J. Han, Xin Jin, J.-A. Ting, S. Vijayakumar, S. Schaal, L. De Raedt, Leave-One-Out Cross-Validation, in: Encycl. Mach. Learn., Springer US, Boston, MA, 2011: pp. 600-601. https://doi.org/10.1007/978-0-387-30164-8_469.

[24] C.W. Holland, D.W. Cravens, Fractional Factorial Experimental Designs in Marketing Research, J. Mark. Res. 10 (1973) 270. https://doi.org/10.2307/3149694.

[25] C. Qi, J. Diao, L. Qiu, IEEE Access, 2019, DOI:10.1109/ACCESS.2019.2892062.

[26] J. Caicedo-Acosta, G. A. Castaño, C. Acosta-Medina, A. Alvarez-Meza, G. Castellanos-Dominguez, Sensors, 2021, DOI:10.3390/s21061932.

[27] X. Yang, L. Li, Q. Tao, W. Lu, M. Li, Comput. Mater. Sci., 2021, DOI:10.1016/j.commatsci.2021.110528.

[28] G. C. Cawley, "Leave-One-Out Cross-Validation Based Model Selection Criteria for Weighted LS-SVMs," The 2006 IEEE International Joint Conference on Neural Network Proceedings, 2006, pp. 1661-1668, doi: 10.1109/IJCNN.2006.246634.

[29] Hawkins, D. M. (2004). The Problem of Overfitting. Journal of Chemical Information and Computer Sciences, 44(1), 1-12. https://doi.org/10.1021/ci0342472

[30] Qi, C., Diao, J., \& Qiu, L. (2019). On Estimating Model in Feature Selection with Cross-Validation. IEEE Access, 7, 33454-33463. https://doi.org/10.1109/ACCESS.2019.2892062

[31] Tan, X., Bi, W., Hou, X., \& Wang, W. (2011). Reliability analysis using radial basis function networks and support vector machines. Computers and Geotechnics, 38(2), 178-186. https://doi.org/10.1016/j.compgeo.2010.11.002 